\title{
On the Supports of Recognizable Timed Series
}

\author{
Karin Quaas \\ Institut für Informatik, Universität Leipzig \\ 04009 Leipzig, Germany \\ quaas@informatik.uni-leipzig.de
}

\begin{abstract}
Recently, the model of weighted timed automata has gained interest within the real-time community. In a previous work, we built a bridge to the theory of weighted automata and introduced a general model of weighted timed automata defined over a semiring and a family of cost functions. In this model, a weighted timed automaton recognizes a timed series, i.e., a function mapping to each timed word a weight taken from the semiring. Continuing in this spirit, the aim of this paper is to investigate the support and cut languages of recognizable timed series. We present results that lead to the decidability of weighted versions of classical decidability problems as e.g. the emptiness problem. Our results may also be used to check whether weighted timed systems satisfy specifications restricting the consumption of a resource.
\end{abstract}

\section{Introduction}

Since its introduction in 2001 by Alur et al. and Behrmann et al. [3, 6], weighted timed automata have become a well-studied object of research within the realtime community. This model allows for a natural modelling of continuous resource consumption of real-time systems and has interesting applications in operations research and optimal scheduling. Weighted timed automata are timed automata [2] extended with one or more variables representing resources e.g. energy, memory or money. A function assigns weights to both the edges and the locations of the timed automaton that may be interpreted as costs or rewards. The variables do not influence the behaviour of a timed automaton, but may be used as a measure of performance. This model gives rise to a lot of interesting questions, e.g. what is the cheapest way to reach a certain location, what are the minimal average costs in non-terminating timed systems, or what is the optimal rate of produced items and energy consumption. Problems of this kind have been thoroughly investigated in recent papers on solving optimal reachability problems $[6,5,3,1,11,9]$, model checking WCTL, WMTL or WLTL $[8,10,14$, $12]$ and weighted timed games $[13,8,10]$.

Recently, we proposed to define weighted timed automata in a general way over a semiring and a family of location cost functions [16]. Doing so, we not only obtain a model that generalizes most of the models used in the literature so far including amongst others the original proposal $[3,6]$ and which may also be used to model weighted timed automata with more than one cost variable 
(as in e.g. [11,19]); but the general definition also gives rise to new instances of models using different semirings and families of cost functions. Moreover, by defining weighted timed automata over semirings, we build a bridge to the wellinvestigated theory of weighted automata. We believe that the connection between weighted automata and weighted timed automata may be cross-fertilizing for both research areas. In particular, while most of the above mentioned papers focus on algorithmic methods that may be used for specific problems in verification, we aim to investigate different aspects of the behaviour of weighted timed automata in terms of timed series, i.e., functions mapping timed words to weights in the semiring. Doing so, we hope to gain a deeper understanding of the nature of weighted timed automata and to build a solid basis for further research on this model. For instance, we were able to generalize two of the most fundamental results in the theory of formal languages to weighted timed automata, namely Kleene's respectively Büchi's theorems on the equality of recognizable and rational respectively MSO-definable languages [16, 21].

Here, we aim to shed light on the so-called supports of timed series, consisting of all timed words which are not mapped to zero. Within the theory of weighted automata, supports have been extensively studied (see eg. [7, 23]). For instance, for large classes of semirings, the support of a recognizable formal power series is known to be recognizable, which implies the decidability of generalizations of some of the fundamental decidability problems of formal language theory as e.g. the emptiness problem or the universality problem. We also want to investigate the recognizability of so-called cut languages. These are sets of timed words which are assigned a weight smaller than (or greater than, respectively) a given value. We believe that both supports and cut languages provide the real-time community with nice applications in the analysis of real-time systems. For instance, we may be interested in whether the set of timed words whose cost under a weighted timed automaton is not exceeding a given value satisfies a specification. Problems like this can be solved using an automata-theoretic approach by using methods presented in this paper.

In this paper, we focus on recognizable timed series over semirings having weights in the reals and families of linear functions, as these allow for the most interesting applications in the theory of weighted timed automata [6, 3, 9]. Many algorithms for decision problems in the (untimed) weighted setting rely on the fact that the set of weights occuring in a weighted automaton is finite. However, in a weighted timed automaton with linear cost functions this is not the case. Thus, the main challenge is to deal with the infinite number of weights occuring in a weighted timed automaton. We can show that for some problems and semirings it is not necessary to consider the exact weights of the transitions participating in a run. Besides linear cost functions, we consider weighted timed automata over step functions for which the problem of infinite weights does not occur. For this kind of functions we can show that most of the results of the (untimed) setting can be carried over to the timed setting. 


\section{Preliminaries}

Let $\mathbb{N}, \mathbb{Z}_{\geq 0}, \mathbb{Q}_{\geq 0}, \mathbb{R}_{\geq 0}$ and $\mathbb{R}$ denote the set of natural, positive integer, positive rational, positive real numbers and real numbers, respectively. We use $\Sigma$ to denote a finite alphabet.

A timed word over $\Sigma$ is a finite sequence $\left(a_{1}, t_{1}\right) \ldots\left(a_{n}, t_{n}\right) \in\left(\Sigma \times \mathbb{R}_{>0}\right)^{*}$, where the sequence of timestamps $t_{1} \ldots t_{n}$ is non-decreasing. We write $T \Sigma^{*}$ for the set of timed words over $\Sigma$. A set $L \subseteq T \Sigma^{*}$ is called a timed language. We say that a timed word is strictly monotonic if its sequence of timestamps is strictly monotonic increasing (i.e., we rule out zero time delays). We use $T_{s} \Sigma^{*}$ to denote the set of strictly monotonic timed words. A timed language $L \subseteq T_{s} \Sigma^{*}$ is called strictly monotonic. Given a timed word $w$ as above we define the length $|w|$ of $w$ to be $n$.

Let $X$ be a finite set of so-called clock variables ranging over $\mathbb{R}_{\geq 0}$. We define clock constraints $\phi$ over $X$ to be conjunctions of formulas of the form $x \sim k$, where $k \in \mathbb{Q}_{\geq 0}, x \in X$, and $\sim \in\{<, \leq,>, \geq\}$. Let $\Phi(X)$ be the set of all clock constraints $\phi$ over $X$. A clock valuation $\nu: X \rightarrow \mathbb{R}_{\geq 0}$ is a function that assigns a value to each clock variable. We let $\nu_{0}$ be the special clock valuation assigning 0 to each clock variable. A clock valuation $\nu$ satisfies a clock constraint $\phi$, written $\nu \models \phi$, if $\phi$ evaluates to true according to the values given by $\nu$. For $\delta \in \mathbb{R}_{\geq 0}$ and $\lambda \subseteq X$, respectively, we define $\nu+\delta$ to be $(\nu+\delta)(x)=\nu(x)+\delta$ for each $x \in X$ and $(\nu[\lambda:=0])(x)=0$ if $x \in \lambda$ and $(\nu[\lambda:=0])(x)=\nu(x)$ otherwise.

A semiring $\mathcal{K}$ is an algebraic structure $(K, \oplus, \odot, \mathbb{O}, \mathbb{1})$ such that $(K, \oplus, \mathbb{O})$ is a commutative monoid, $(K, \odot, \mathbb{1})$ is a monoid, $\odot$ distributes over $\oplus$, and $\mathbb{O}$ is absorbing. As an example, consider the min-plus-semiring $\left(\mathbb{R}_{\geq 0} \cup\{\infty\}, \min ,+, \infty, 0\right)$ or the semiring $(\mathbb{R},+, \cdot, 0,1)$ over the reals with ordinary addition and multiplication. Let $\Gamma$ be a set. A mapping $\mathcal{T}: \Gamma^{*} \rightarrow K$ is called a series. For historical reasons, we write $(\mathcal{T}, w)$ instead of $\mathcal{T}(w)$ for $w \in \Gamma^{*}$. The support $\operatorname{supp}(\mathcal{T})$ of $\mathcal{T}$ is defined to be the set $\left\{w \in \Gamma^{*} \mid(\mathcal{T}, w) \neq \mathbb{O}\right\}$.

We let $\mathcal{F}$ be a family of functions from $\mathbb{R}_{\geq 0}$ to $K$. In this paper, we are mainly interested in two kinds of such families, namely the family of step functions and the family of linear functions. A function $f: \mathbb{R}_{>0} \rightarrow K$ is a step function if it is of the form $f(\delta)=\bigoplus_{1 \leq i \leq n} \alpha_{i} \odot \chi_{A_{i}}(\delta)$ for every $\delta \in \mathbb{R}_{\geq 0}$, where $n \in \mathbb{N}, \alpha_{i} \in K$, $A_{i}$ are intervals over $\mathbb{R}_{\geq 0}$ with borders in $\mathbb{Q}_{\geq 0}$ such that $A_{j} \cap A_{k}=\emptyset$ for $j \neq k$ and $\bigcup_{1<i<n} A_{i}=\mathbb{R}_{\geq 0}$, and $\chi_{A_{i}}$ is a characteristic function of $A_{i}$, i.e., we have $\chi_{A_{i}}(\delta)=\mathbb{1}$ if $\delta \in A_{i}$ and $\mathbb{O}$ otherwise, for every $i \in\{1, \ldots, n\}$. A step function has the important property of having a finite image. For semirings where $K \supseteq \mathbb{R}>0$, we say that a function $f: \mathbb{R}_{\geq 0} \rightarrow K$ is linear if it is of the form $f(\delta)=k \cdot \delta$ for every $\delta \in \mathbb{R}_{\geq 0}$ and some $k \in K$.

A weighted timed automaton over $\mathcal{K}, \Sigma$ and $\mathcal{F}$ is a tuple $\left(S, S_{0}, S_{f}, X, E, C\right)$, where

$-S$ is a finite set of locations (states)

$-S_{0} \subseteq S$ is a set of initial locations

$-S_{f} \subseteq S$ is a set of final locations

$-X$ is a finite set of clock variables 
- $E \subseteq S \times \Sigma \times \Phi(X) \times 2^{X} \times S$ is a finite set of edges. An edge $\left(s, \sigma, \phi, \lambda, s^{\prime}\right)$ allows a jump from $s$ to $s^{\prime}$ if $\sigma$ is read, provided that for the current clock valuation $\nu$ we have $\nu \models \phi$. After the edge has been executed, the new clock valuation is $\nu[\lambda:=0]$.

$-C=\left\{C_{\mathcal{E}}\right\} \cup\left\{C_{s}: s \in S\right\}$, where $C_{\mathcal{E}}: E \rightarrow K$, and $C_{s} \in \mathcal{F}$ for any $s \in S$.

A weighted timed automaton $\mathcal{A}$ induces an infinite state transition system consisting of states of the form $(s, \nu)$, where $s \in S$ and $\nu$ is a clock valuation. Between the states there are timed transitions of the form $(s, \nu) \stackrel{\delta}{\longrightarrow}(s, \nu+\delta)$ for some $\delta \in \mathbb{R}_{\geq 0}$, and discrete transitions of the form $(s, \nu) \stackrel{e}{\longrightarrow}\left(s^{\prime}, \nu^{\prime}\right)$ for some $e=\left(s, a, \bar{\phi}, \lambda, s^{\prime}\right) \in E$ such that $\nu \models \phi$ and $\nu^{\prime}=\nu[\lambda:=0]$. A transition is a timed transition $(s, \nu) \stackrel{\delta}{\longrightarrow}(s, \nu+\delta)$ followed by a discrete transition $(s, \nu+\delta) \stackrel{e}{\longrightarrow}\left(s^{\prime}, \nu^{\prime}\right)$, abbreviated by $(s, \nu) \stackrel{\delta}{\longrightarrow} \stackrel{e}{\longrightarrow}\left(s^{\prime}, \nu^{\prime}\right)$. A run of $\mathcal{A}$ on a timed word $w$ is a finite sequence $\left(s_{0}, \nu_{0}\right) \stackrel{\delta_{1}}{\longrightarrow} \stackrel{e_{1}}{\longrightarrow}\left(s_{1}, \nu_{1}\right) \stackrel{\delta_{2}}{\longrightarrow} \stackrel{e_{2}}{\longrightarrow} \ldots \stackrel{\delta_{|w|}}{\longrightarrow} \stackrel{e_{|w|}}{\longrightarrow}\left(s_{|w|}, \nu_{|w|}\right)$ of transitions, where $\nu_{0}=0^{X}$. We say that a run $r$ is successful if $s_{0} \in S_{0}$ and $s_{|w|} \in S_{f}$. We define the running weight $\operatorname{rwt}(r)$ of a run $r$ as above to be $\bigodot_{1 \leq i \leq|w|} C_{s_{i-1}}\left(\delta_{i}\right) \odot C_{\mathcal{E}}\left(e_{i}\right)$. The behaviour $\|\mathcal{A}\|: T \Sigma^{*} \rightarrow K$ of $\mathcal{A}$ is given by $(\|\mathcal{A}\|, w)=\bigoplus\{\operatorname{rwt}(r): r$ is a successful run of $\mathcal{A}$ on $w\}$. A function $\mathcal{T}: T \Sigma^{*} \rightarrow K$ is called a timed series. A timed series $\mathcal{T}$ is recognizable over $\mathcal{K}, \Sigma$ and $\mathcal{F}$ if there is a weighted timed automaton $\mathcal{A}$ over $\mathcal{K}, \Sigma$ and $\mathcal{F}$ with $\|\mathcal{A}\|=\mathcal{T}$.

An (unweighted) timed automaton as defined by Alur and Dill [2] can be seen as weighted timed automaton over the Boolean semiring and the family of constant functions, where $C$ maps every edge and every pair in $S \times \mathbb{R}_{\geq 0}$ to $\mathbb{1}$. In this case the behaviour of the automaton $\mathcal{A}$ is a characteristic function and we have $L(\mathcal{A})=\operatorname{supp}(\|\mathcal{A}\|)$. If we ignore all timing information and let $C$ map every pair in $S \times \mathbb{R}_{\geq 0}$ to $\mathbb{1}$, we obtain the model of (untimed) weighted automata. The standard model of weighted timed automata $[3,6]$ can be modeled using the min-plus-semiring and the family of linear functions.

A (weighted) timed automaton is unambiguous, if for every timed word $w$ there is at most one successful run. A timed language (timed series, respectively) is called unambiguously recognizable, if there is an unambiguous timed automaton (weighted timed automaton, respectively) recognizing it.

A timed series $\mathcal{T}$ is called strictly monotonic, if for each timed word $w \in$ $T \Sigma^{*} \backslash T_{s} \Sigma^{*}$ we have $(\mathcal{T}, w)=\mathbb{0}$.

Given a weighted timed automaton $\mathcal{A}=\left(S, S_{0}, S_{f}, X, E, C\right)$, we define wgt $_{e}(\mathcal{A})=\left\{C_{\mathcal{E}}(e) \mid e \in E\right\}$ and $\operatorname{wgt}_{s}(\mathcal{A})=\left\{C_{s}(\delta) \mid s \in S, \delta \in \mathbb{R}_{\geq 0}\right\}$ and put $\operatorname{wgt}(\mathcal{A})=w_{g t}(\mathcal{A}) \cup w_{g t}(\mathcal{A})$. Let $\mathcal{I}$ be the set of all intervals over $\mathbb{R}_{\geq 0}$ with borders in $\mathbb{Q}_{\geq 0}$ and $x$ a clock variable. We define a function \langle\rangle$_{x}: \mathcal{I} \rightarrow \Phi(\{x\})$ by $\mathrm{s}\langle I\rangle_{x}=\left\{\begin{array}{ll}a<x \wedge x<b & \text { if } I=(a, b) \\ a \leq x \wedge x<b & \text { if } I=[a, b) \\ a<x \wedge x \leq b & \text { if } I=(a, b] \\ a \leq x \wedge x \leq b & \text { if } I=[a, b]\end{array}\right.$ for every $I \in \mathcal{I}$. 


\section{Supports of Recognizable Timed Series}

In this section, we study whether the support of a recognizable timed series is a recognizable timed language. It is well-known that in the untimed setting the support of every series recognizable over a positive semiring is recognizable [24]. A semiring is positive if it is both zero-sum free and zero-divisor free, i.e., we have $k \oplus k^{\prime}=\mathbb{O}$ implies $k=\mathbb{O}$ and $k^{\prime}=\mathbb{O}$, and $k \odot k^{\prime}=\mathbb{O}$ implies $k=\mathbb{O}$ or $k^{\prime}=\mathbb{0}$ for each $k, k^{\prime} \in K$. With these two properties it is easy to construct a finite automaton recognizing exactly the support of the behaviour of some given weighted automaton by simply removing all edges with weight $\mathbb{0}$ [24].

For the timed setting, this idea can more or less be adopted by removing not only the edges with weight $\mathbb{O}$ but also all locations whose cost functions map all time delays to $\mathbb{0}$. However, for certain positive semirings and families the method does only work for strictly monotonic timed series. For instance, let $\mathcal{K}=\left(\mathbb{R}_{\geq 0},+, \cdot, 0,1\right)$ and $\mathcal{F}$ be the family of linear functions. Even if in a weighted timed automaton over $\mathcal{K}$ and $\mathcal{F}$ we remove all locations with cost functions of the form $f(\delta)=0 \cdot \delta$, we cannot conclude that in the resulting weighted timed automaton every successful run has a running weight different from 0 . This is because we have $f(0)=0$ for every $f \in \mathcal{F}$ and thus the running weight of every successful run on a timed word with a zero delay equals 0 .

Lemma 1. Let $\mathcal{K}$ be positive.

1. Assume that for all $f \in \mathcal{F}$ we have either $f(\delta) \neq \mathbb{0}$ for all $\delta \in \mathbb{R}_{\geq 0}$ or $f(\delta)=\mathbb{0}$ for all $\delta \in \mathbb{R}_{\geq 0}$. If $\mathcal{T}$ is a recognizable timed series, then $\operatorname{supp}(\mathcal{T})$ is recognizable.

2. Assume that for all $f \in \mathcal{F}$ we have either $f(\delta) \neq \mathbb{0}$ for all $\delta \in \mathbb{R}_{\geq 0} \backslash\{0\}$ or $f(\delta)=\mathbb{O}$ for all $\delta \in \mathbb{R}_{\geq 0} \backslash\{0\}$. If $\mathcal{T}$ is a strictly monotonic and recognizable timed series, then $\operatorname{supp}(\mathcal{T})$ is strictly monotonic and recognizable.

As a consequence, the support of the behaviour of each weighted timed automaton over the widely-used $[6,3,9]$ setting of the min-plus-semiring and the family of linear functions is recognizable. To give another example, the first part of Lemma 1 also applies to the semiring $([0,1], \max , \cdot, 0,1)$ and families of functions of the form $f(\delta)=k^{\delta}$ for some $k \in[0,1]$ and each $\delta \in \mathbb{R}_{\geq 0}$.

In the past few years, weighted timed automata with multiple prices have attracted interest $[11,19]$. These may be modeled using the direct product of e.g. the min-plus-semiring and component-wisely defined linear functions. Unfortunately, while still being zero-sum free, direct products of positive semirings are not zero-divisor free and thus Lemma 1 cannot be applied. Very recently, Kirsten showed that the commutativity and zero-sum freeness of the semiring is a sufficient condition for the support of a recognizable series being recognizable [18]. Kirsten's proof method strongly relies on the fact that the set of disjoint weights occurring in any of the runs of the weighted automaton is finite. However, for weighted timed automata over the family of linear functions this is not the case. Nevertheless, for certain semirings we can adapt the proof by exploiting the fact that the exact weights emerging from staying in a location are not crucial for 
deciding whether the running weight of a run equals $\mathbb{O}$ or not, but rather it is sufficient to consider the semiring coefficients that the time delay is multiplied with, as the following example shows.

Example 1. Let $\mathcal{A}$ be a weighted timed automaton over the min-plus-semiring. For the sake of simplicity, in this example we assume that $C_{\mathcal{E}}(e)=0$ for every edge $e$. Then for each run $r$ of $\mathcal{A}$ on a timed word $w$ we have $\operatorname{rwt}(r)=\infty$ iff there is some $i \in\{1, \ldots,|w|\}$ such that $C_{s_{i-1}}\left(\delta_{i}\right)=\infty$. However, for every location $s$ and time delay $\delta$, we have $C_{s}(\delta)=\infty$ iff $C_{s}(\delta)=\infty \cdot \delta$ for each $\delta \in \mathbb{R}_{\geq 0}$. Hence, the exact time delays are not important but it is sufficient to consider the coefficients of the cost functions of the participating locations in a run. For the direct product of the min-plus-semiring we have a similar result: $\operatorname{rwt}(r)=(\infty, \infty)$ iff either there is some $i \in\{1, \ldots,|w|\}$ such that $C_{s_{i-1}}\left(\delta_{i}\right)=$ $(\infty, \infty)$, or there are $i, j \in\{1, \ldots,|w|\}$ such that $i \neq j$ and $C_{s_{i-1}}\left(\delta_{i}\right)=(a, \infty)$ and $C_{s_{j-1}}\left(\delta_{j}\right)=(\infty, b)$, for some $a, b \in \mathbb{R}_{\geq 0}$. Again, the exact time delays are not important.

This leads us to the following result.

Lemma 2. Let $\mathcal{K}$ be commutative and zero-sum free.

1. If $\mathcal{F}$ is the family of step functions and $\mathcal{T}$ is a recognizable timed series, then $\operatorname{supp}(\mathcal{T})$ is recognizable.

2. If $\mathcal{F}$ is the family of linear functions and $\mathcal{K}$ is (the direct product of) one of the following semirings

(a) $\left(\mathbb{R}_{\geq 0} \cup\{\infty\}, \min ,+, \infty, 0\right)$

(b) $\left(\mathbb{R}_{\geq 0} \cup\{-\infty\}\right.$, $\left.\max ,+,-\infty, 0\right)$

(c) $\left(\mathbb{R}_{\geq 0} \cup\{\infty,-\infty\}\right.$, min, max, $\left.\infty,-\infty\right)$

and $\mathcal{T}$ is a recognizable timed series, then $\operatorname{supp}(\mathcal{T})$ is recognizable.

3. If $\mathcal{F}$ is the family of linear functions, $\mathcal{K}$ is the (direct product of) $\left(\mathbb{R}_{\geq 0},+, \cdot, 0,1\right)$, and $\mathcal{T}$ is strictly monotonic and recognizable, then $\operatorname{supp}(\mathcal{T})$ is strictly monotonic and recognizable.

None of the constructions used in the proofs of Lemmas 1 and 2 increases the number of clock variables used. Combining these two results with the decidability of the emptiness problem for timed automata [2] and the universality problem for single-clock timed automata [20], we obtain the following corollary.

Corollary 1. If $\mathcal{K}, \mathcal{F}$ and $\mathcal{T}$ correspond to one of the settings defined in Lemmas 1 and 2 , then it is decidable whether $\operatorname{supp}(\mathcal{T})=\emptyset$. If, additionally, $\mathcal{T}$ can be recognized by a single-clock weighted timed automaton, then it is decidable whether $\operatorname{supp}(\mathcal{T})=T \Sigma^{*}$.

Next, we consider recognizable timed series over fields, i.e., semirings where $(K, \oplus, \mathbb{O})$ is a group and $(K \backslash\{\mathbb{O}\}, \odot, \mathbb{1})$ is a commutative group. It is well-known that already in the untimed setting there are recognizable series over fields for which the support is not recognizable (see e.g. [18]). Yet, by a result of Berstel and Reutenauer [7], it is decidable whether the support of a recognizable series over a field is empty or not. In the next section, we show that this also holds for recognizable timed series over certain fields and families of cost functions. 


\section{The Empty Support Problem for Fields}

In the following, we let $\mathcal{K}$ be a field, e.g. the semiring over the rationals or reals with the usual addition and multiplication operations. We start to show that for this class of semirings and the family of step functions, it is decidable whether the support of a given recognizable timed series $\mathcal{T}$ is empty or not. The idea is to reduce the problem for recognizable timed series to the corresponding classical problem for recognizable (untimed) series, which is known to be decidable [7].

Lemma 3. Let $\mathcal{K}$ be a field and $\mathcal{F}$ be the family of step functions. If $\mathcal{T}: T \Sigma^{*} \rightarrow$ $K$ is a recognizable timed series over $\mathcal{K}$ and $\mathcal{F}$, then there is a recognizable series $\mathcal{T}^{\prime}: \Sigma^{*} \rightarrow K$ over $\mathcal{K}$ such that $\operatorname{supp}\left(\mathcal{T}^{\prime}\right)=\emptyset$ iff $\operatorname{supp}(\mathcal{T})=\emptyset$.

Proof. Let $\mathcal{A}$ be a weighted timed automaton such that $\|\mathcal{A}\|=\mathcal{T}$. Let $R(\mathcal{A})$ be the region automaton of the timed automaton underlying $\mathcal{A}[2]$. We add a cost function $\mu$ to $R(\mathcal{A})$, assigning weights taken from $K$ to every transition in $R(\mathcal{A})$, obtaining a weighted automaton over $\mathcal{K}$ which we also will denote by $R(\mathcal{A})$. The cost function $\mu$ is defined as follows: if $t$ is a transition that stems from an edge $e \in E$ in $\mathcal{A}$, we put $\mu(t)=C_{\mathcal{E}}(e)$. Now, let $t$ be a transition that stems from a timed transition labelled with $\delta$ starting in some location $s$, and assume $C_{s}\left(\delta^{\prime}\right)=\bigoplus_{1 \leq i \leq n} \alpha_{i} \odot \chi_{A_{i}}\left(\delta^{\prime}\right)$ for every $\delta^{\prime} \in \mathbb{R}_{\geq 0}$. There is a unique $i \in\{1, \ldots, n\}$ such that $\delta \in A_{i}$. We define $\mu(t)=\alpha_{i}$. Using this and the fact that the infinite state-transition system induced by the timed automaton underlying $\mathcal{A}$ and the region automaton $R(\mathcal{A})$ are bisimulation equivalent [2], one can easily show that there is a weight-preserving bijective correspondence between the set of successful runs of $\mathcal{A}$ and $R(\mathcal{A})$. This implies $\operatorname{supp}(\|\mathcal{A}\|)=\emptyset$ iff $\operatorname{supp}(\|R(\mathcal{A})\|)=\emptyset$.

Now we want to consider weighted timed automata over fields and the family of linear functions. Clearly, due to the infinite number of weights occuring in this kind of automata, we cannot use the same construction as above. However, similarly to the case of non-zero-divisor free semirings in Sect.3, for certain semirings we do not need to consider the exact weight of a transition in order to decide whether a running weight equals $\mathbb{O}$ or not.

Lemma 4. Let $\mathcal{K}=(\mathbb{R},+, \cdot, 0,1)$ and $\mathcal{F}$ be the family of linear functions. If $\mathcal{T}: T \Sigma^{*} \rightarrow K$ is a recognizable timed series over $\mathcal{K}$ and $\mathcal{F}$, then there is a recognizable series $\mathcal{T}^{\prime}: \Sigma^{*} \rightarrow K$ over $\mathcal{K}$ such that $\operatorname{supp}\left(\mathcal{T}^{\prime}\right)=\emptyset$ iff $\operatorname{supp}(\mathcal{T})=\emptyset$.

Proof. Let $\mathcal{A}$ be a weighted timed automaton such that $\|\mathcal{A}\|=\mathcal{T}$. Again, we extend the region automaton $R(\mathcal{A})$ of the timed automaton underlying $\mathcal{A}$ with a cost function $\mu$, obtaining a weighted automaton over $\mathcal{K}$ which we also will denote by $R(\mathcal{A})$. This time, the cost function $\mu$ is defined as follows: if $t$ is a transition that stems from an edge $e \in E$ in $\mathcal{A}$, we put $\mu(t)=C_{\mathcal{E}}(e)$. Now, let $t$ be a transition that stems from a timed transition labelled with $\delta$ starting in some location $s$, and assume $C_{s}\left(\delta^{\prime}\right)=c_{s} \cdot \delta^{\prime}$ for every $\delta^{\prime} \in \mathbb{R}_{\geq 0}$. We define $\mu(t)=c_{s}$ if $\delta \neq 0, \mu(t)=0$ otherwise. By the fact that the infinite statetransition system induced by the timed automaton underlying $\mathcal{A}$ and the region 
automaton $R(\mathcal{A})$ are bisimulation equivalent [2], it follows that there is a bijective correspondence between the set of successful runs of $\mathcal{A}$ and $R(\mathcal{A})$; however, this correspondence is not weight-preserving. Let $w \in T \Sigma^{*}$. We use $\operatorname{abs}(w)$ to denote the unique (untimed) word labelling the runs of $R(\mathcal{A})$ corresponding to runs of $\mathcal{A}$ on $w$. We show that $(\|\mathcal{A}\|, w)=0$ implies $(\|R(\mathcal{A})\|, \operatorname{abs}(w))=0$. Let $r=\left(s_{0}, \nu_{0}\right) \stackrel{\delta_{1}}{\longrightarrow} \stackrel{e_{1}}{\longrightarrow}\left(s_{1}, \nu_{1}\right) \stackrel{\delta_{2}}{\longrightarrow} \stackrel{e_{2}}{\longrightarrow} \ldots \stackrel{\delta_{|w|}}{\longrightarrow} \stackrel{e_{|w|}}{\longrightarrow}\left(s_{|w|}, \nu_{|w|}\right)$ be a successful run of $\mathcal{A}$ on $w$. Using commutativity of - and assuming every location cost function $C_{s}$ in $\mathcal{A}$ to be of the form $C_{s}(\delta)=c_{s} \cdot \delta$ for every $\delta \in \mathbb{R}_{\geq 0}$, we obtain

$$
\operatorname{rwt}(r)=\prod_{1 \leq i \leq|w|} c_{s_{i-1}} \cdot C_{\mathcal{E}}\left(e_{i}\right) \cdot \prod_{1 \leq i \leq|w|} \delta_{i}
$$

Let $n$ be the (finite) number of successful runs of $\mathcal{A}$ on $w$ and let $r^{i}$ be a successful run of $\mathcal{A}$ on $w$ for each $i \in\{1, \ldots, n\}$. Then, by distributivity, we have

$$
\begin{aligned}
(\|\mathcal{A}\|, w) & =\sum_{1 \leq i \leq n} \operatorname{rwt}\left(r^{i}\right) \\
& =\sum_{1 \leq i \leq n}\left[\left(\prod_{1 \leq j \leq|w|} c_{s_{j-1}}^{i} \cdot C_{\mathcal{E}}\left(e_{j}^{i}\right)\right) \cdot \prod_{1 \leq j \leq|w|} \delta_{j}\right] \\
& =\left(\sum_{1 \leq i \leq n} \prod_{1 \leq j \leq|w|} c_{s_{j-1}}^{i} \cdot C_{\mathcal{E}}\left(e_{j}^{i}\right)\right) \cdot \prod_{1 \leq j \leq|w|} \delta_{j} .
\end{aligned}
$$

Clearly, $(\|\mathcal{A}\|, w)=0$ iff $\sum_{1 \leq i \leq n} \prod_{1 \leq j \leq|w|} c_{s_{j-1}}^{i} \cdot C_{\mathcal{E}}\left(e_{j}^{i}\right)=0$ or $\prod_{1 \leq j \leq|w|} \delta_{j}=0$. In the latter case, there is some $j \in\{1, \ldots,|w|\}$ such that $\delta_{j}=0$. Then, the weight $\mu(t)$ of the transition that stems from the timed transition labelled with $\delta_{j}$ equals 0 . Hence, for each $i \in\{1, \ldots, n\}$, the running weight of the successful run of $R(\mathcal{A})$ corresponding to $r^{i}$ equals 0 and we have $(\|R(\mathcal{A})\|$, abs $(w))=0$. Now, assume that for every $1 \leq j \leq|w|$ we have $\delta_{j}>0$ and $\sum_{1 \leq i \leq n} \prod_{1 \leq j \leq|w|} c_{s_{j-1}}^{i}$. $C_{\mathcal{E}}\left(e_{j}^{i}\right)=0$. The definition of $\mu$ for this case implies that the running weights of the corresponding runs in $R(\mathcal{A})$ equal $\prod_{1 \leq j \leq|w|} c_{s_{j-1}}^{i} \cdot C_{\mathcal{E}}\left(e_{j}^{i}\right)$ for each $1 \leq$ $i \leq n$ and thus we have $(\|R(\mathcal{A})\|, a b s(w))=0$. Hence, if $\operatorname{supp}(\|\mathcal{A}\|)=\emptyset$ then $\operatorname{supp}(\|R(\mathcal{A})\|)=\emptyset$. The proof for the other direction can be done analogously.

Observe that the crucial point for obtaining the result is the fact that the multiplication of the semiring coincides with the multiplication used for the definitions of linear functions. The proof may be extended to other semirings and families of functions for which an analogous property holds, i.e., where we use functions of the form $k \odot \delta$ for some $k \in K$. Also notice that - as opposed to the second part of Lemma 1 - we do not need to restrict the application of Lemma 4 to strictly monotonic timed series. As a direct consequence, the following corollary (based on the fact that for every recognizable series over a field it is decidable whether its support is empty or not [7]) also holds for non-strictly monotonic timed series 
over $\left(\mathbb{R}_{\geq 0},+, \cdot, 0,1\right)$ and families of linear functions (generalizing the first part of Corollary 1 for this setting).

Corollary 2. If $\mathcal{K}, \mathcal{F}$ and $\mathcal{T}$ correspond to one of the settings defined in Lemmas 3 and 4 , then it is decidable whether $\operatorname{supp}(\mathcal{T})=\emptyset$.

Remark 1. For the class of recognizable series over fields it is not decidable whether the support equals $\Sigma^{*}[7]$. Hence, we cannot use a reduction as above to solve the universal support problem for timed series recognizable by singleclock weighted timed automata.

\section{Timed Cut Languages}

In this section, we are interested in those timed words whose weights under a timed series exactly correspond to a given value, or whose weights do (not) exceed a given value from the semiring. Sets of words of the second category are knows as cut languages and play an important role in the theory of weighted automata (see eg. $[17,22]$ ). In particular, we want to investigate the following problems: given a recognizable timed series $\mathcal{T}$ and $k \in K$, are the sets

$$
\begin{aligned}
& -\mathcal{T}^{-1}(k)=\left\{w \in T \Sigma^{*} \mid(\mathcal{T}, w)=k\right\} \\
& -\left\{w \in T \Sigma^{*} \mid(\mathcal{T}, w) \leq k\right\} \\
& -\left\{w \in T \Sigma^{*} \mid(\mathcal{T}, w) \geq k\right\}
\end{aligned}
$$

recognizable? Besides new applications in the analysis of real-time systems, a solution to the questions above can also help to solve problems from the theory of timed languages, as the following example (adopted from [17]) shows: assume there is some semiring $\mathcal{K}$ and a family $\mathcal{F}$ such that there is some $f \in \mathcal{F}$ with $f(\delta)=\mathbb{1}$ for each $\delta \in \mathbb{R}_{\geq 0}$ and we can show that $\mathcal{T}^{-1}(k)$ is a recognizable timed language for every recognizable timed series $\mathcal{T}$ and every $k \in K$. Then, we could conclude that the complement $\bar{L}$ of every unambiguously recognizable timed language $L$ is recognizable. The proof of this is as follows: let $L$ be an unambiguously recognizable timed language and $\mathcal{A}$ be an unambiguous timed automaton such that $L(\mathcal{A})=L$. Then, simply assign the weight $\mathbb{1}$ to all edges of $\mathcal{A}$ and let $C_{s}=f$ for any location $s$. Clearly, the behaviour $\left\|\mathcal{A}^{\prime}\right\|$ of the resulting weighted timed automaton $\mathcal{A}^{\prime}$ corresponds to the characteristic function of $L$. But then by assumption the timed language $\left\|\mathcal{A}^{\prime}\right\|^{-1}(\mathbb{O})=\bar{L}$ must be recognizable. However, by now it is not known whether negation preserves recognizability of unambiguous timed languages or not [25].

A semiring $\mathcal{K}$ has characteristic zero if there is no $n \in \mathbb{N} \backslash\{0\}$ such that $\underbrace{\mathbb{1} \oplus \ldots \oplus \mathbb{1}}_{n}=\mathbb{0}$.

Lemma 5. Let $\mathcal{K}$ have characteristic zero and assume that there is some $f \in \mathcal{F}$ such that $f(\delta)=\mathbb{1}$ for each $\delta \in \mathbb{R}_{\geq 0}$. Then there is some recognizable timed series $\mathcal{T}$ over $\mathcal{K}$ and $\mathcal{F}$ such that $\mathcal{T}^{-1}(\mathbb{O})$ is not recognizable. 
Proof. It is well-known that the class of recognizable timed languages is not closed under complement [2]. So let $L \subseteq T \Sigma^{*}$ be a recognizable timed language such that the complement $\bar{L}$ is not recognizable (e.g. the example language in Theorem 1 in [4]). We further let $\mathcal{A}$ be a timed automaton recognizing $L$. We obtain a weighted timed automaton $\mathcal{A}^{\prime}$ over $\mathcal{K}$ and $\mathcal{F}$ by adding a cost function $C$ such that $\mathbb{1}$ is assigned to every edge and the cost function of every location is $f$. Let $w \in L$. Then the running weight of every successful run of $\mathcal{A}^{\prime}$ on $w$ is $\mathbb{1}$. Exploiting the condition on $\mathcal{K}$, we get $\left(\left\|\mathcal{A}^{\prime}\right\|, w\right) \neq \mathbb{0}$. Hence, $L$ is the support of the behaviour of $\mathcal{A}^{\prime}$, formally $\operatorname{supp}\left(\left\|\mathcal{A}^{\prime}\right\|\right)=L$. But this implies $\|\mathcal{A}\|^{-1}(\mathbb{O})=\bar{L}$, which by assumption is not recognizable.

The condition on the semiring is satisfied by every semiring which is not a ring but also e.g. by the semiring over the integers with natural addition and multiplication. In particular, given a recognizable timed series $\mathcal{T}$ over the minplus-semiring (max-plus-semiring, respectively) and the family of step functions or linear functions, in general $\mathcal{T}^{-1}(\mathbb{O})$ is not recognizable. In contrast to this, the lemma cannot be applied if $\mathcal{T}$ is recognizable over the semiring $\left(\mathbb{R}_{\geq 0},+, \cdot, 0,1\right)$ and the family $\mathcal{F}$ of linear functions, as there is no function $f \in \mathcal{F}$ satisfying $f(\delta)=1$ for every $\delta \in \mathbb{R}_{\geq 0}$. Notice that for the family of step functions there is such a function. This raises the interesting question whether in general, assuming that $\mathcal{K}$ is fixed, we cannot conclude from a "negative" result for the family of step functions that a negative result also holds for the (more expressive and harder) family of linear functions.

The negative result in Lemma 5 mainly relies on the non-closure of recognizable timed languages under complement. The question arises whether we can obtain a positive result for $k \neq \mathbb{O}$. Unfortunately, for the most interesting setting of real-valued weights and linear cost functions we have to give a negative answer, even if we confine our study to unambiguously recognizable timed series.

Lemma 6. Let $\mathcal{K}$ be one of the following semirings

$$
\begin{aligned}
& -\left(\mathbb{R}_{\geq 0} \cup\{\infty\}, \min ,+, \infty, 0\right) \\
& -\left(\mathbb{R}_{\geq 0} \cup\{-\infty\}, \max ,+,-\infty, 0\right) \\
& -\left(\mathbb{R}_{,+,}+, 0,1\right) \\
& -\left(\mathbb{R}_{\geq 0},+, \cdot, 0,1\right)
\end{aligned}
$$

and $\mathcal{F}$ be the family of linear functions. Then there is some unambiguously recognizable timed series $\mathcal{T}$ over $\mathcal{K}$ and $\mathcal{F}$ and some $k \in K \backslash\{\mathbb{0}\}$ such that $\mathcal{T}^{-1}(k)$ is not recognizable.

Proof. We show the proof for $\left(\mathbb{R}_{\geq 0},+, \cdot, 0,1\right)$. The proofs for the other semirings can be done analogously. Let $\mathcal{A}$ be the unambiguous weighted timed automaton over $\left(\mathbb{R}_{\geq 0},+, \cdot, 0,1\right)$ and the family of linear functions shown in the figure below. Further, we put $k=6$. Assume there is a timed automaton $\mathcal{A}^{\prime}$ such that $L\left(\mathcal{A}^{\prime}\right)=$ $\|\mathcal{A}\|^{-1}(k)$. Let $w=\left(a, t_{1}\right)\left(a, t_{2}\right)$ be some timed word such that $(\|\mathcal{A}\|, w)=6$, e.g. $(a, 1.5)(a, 0.3)$. 


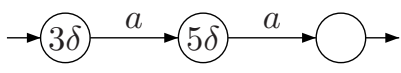

Hence, there must be a successful run $r$ of $\mathcal{A}^{\prime}$ on $w$. Choose an arbitrary $\epsilon$ such that $t_{2}+\epsilon \in \mathbb{R}_{\geq 0} \backslash \mathbb{Q}_{\geq 0}$ and let $w^{\prime}=\left(a, t_{1}\right)\left(a, t_{2}+\epsilon\right)$. Then, the discrete transitions of $r$ must also constitute a successful run of $\mathcal{A}^{\prime}$ on $w^{\prime}$, because the constants in a guard may only be rational numbers and hence $\mathcal{A}^{\prime}$ cannot distinguish between the time delays $t_{2}-t_{1}$ and $\left(t_{2}+\epsilon\right)-t_{1}$. Hence, $w^{\prime} \in L\left(\mathcal{A}^{\prime}\right)$, but $\left(\|\mathcal{A}\|, w^{\prime}\right)>6$.

Next, we show that if we consider unambiguously recognizable timed series over the family of step functions, we can give a positive answer. This may be not too surprising, as in this case the number of weights occuring in a weighted timed automaton is finite and we can apply proof methods known from the theory of weighted automata (see e.g. [17]). For the sake of completeness we present some of the results in the following. These may also serve as a starting point for further research on other kinds of cost functions.

A morphism between semirings $\left(K, \oplus, \odot, \mathbb{0}_{K}, \mathbb{1}_{K}\right)$ and $\left(K^{\prime}, \oplus^{\prime}, \odot^{\prime}, \mathbb{D}_{K^{\prime}}, \mathbb{1}_{K^{\prime}}\right)$ is a function $\eta: K \rightarrow K^{\prime}$ satisfying

- $\eta(a \oplus b)=\eta(a) \oplus^{\prime} \eta(b)$ for all $a, b \in K$

- $\eta(a \odot b)=\eta(a) \odot^{\prime} \eta(b)$ for all $a, b \in K$

- $\eta\left(\mathbb{O}_{K}\right)=\mathbb{0}_{K^{\prime}}$ and $\eta\left(\mathbb{1}_{K}\right)=\mathbb{1}_{K^{\prime}}$.

Lemma 7. Let $\mathcal{K}, \mathcal{K}^{\prime}$ be two semirings and $\mathcal{F}$ be the family of step functions from $\mathbb{R}_{\geq 0}$ to $K$. If $\mathcal{T}$ is a recognizable timed series over $\mathcal{K}$ and $\mathcal{F}$ and $\eta: \mathcal{K} \rightarrow \mathcal{K}^{\prime}$ is a semiring morphism, then $\eta \circ \mathcal{T}$ is recognizable over $\mathcal{K}^{\prime}$ and $\mathcal{F}$.

Proof. If $\mathcal{A}$ is the weighted timed automaton over $\mathcal{K}$ recognizing $\mathcal{T}$, we obtain a weighted timed automaton $\mathcal{A}^{\prime}$ over $\mathcal{K}^{\prime}$ by replacing all constants $k \in K$ occuring in the cost functions of $\mathcal{A}$ by $\eta(k)$. Then one can show that there is a bijective correspondence between the set of successful runs $r$ of $\mathcal{A}$ and the set of successful runs $r^{\prime}$ of $\mathcal{A}^{\prime}$ such that $\operatorname{rwt}\left(r^{\prime}\right)=\eta(\operatorname{rwt}(r))$. Thus, we have

$$
\begin{aligned}
\left(\left\|\mathcal{A}^{\prime}\right\|, w\right) & =\bigoplus\left\{\operatorname{rwt}\left(r^{\prime}\right) \mid r^{\prime} \text { is a successful run of } \mathcal{A}^{\prime} \text { on } w\right\} \\
& =\bigoplus\{\eta(\operatorname{rwt}(r)) \mid r \text { is a successful run of } \mathcal{A} \text { on } w\} \\
& =\eta(\bigoplus\{\operatorname{rwt}(r) \mid r \text { is a successful run of } \mathcal{A} \text { on } w\}) \\
& =\eta(\|\mathcal{A}\|, w)
\end{aligned}
$$

Lemma 8. Let $\mathcal{F}$ be the family of step functions, $k \in K \backslash\{\mathbb{0}\}$ and $\mathcal{T}$ be an unambiguously recognizable timed series. If $\mathcal{K}$ is (the direct product of) one of the following semirings

1. $(K \cup\{\infty\}, \min ,+, \infty, 0)$ for $K \in\left\{\mathbb{N}, \mathbb{Z}_{\geq 0}, \mathbb{Q}_{\geq 0}, \mathbb{R}_{\geq 0}\right\}$, 
2. $(\mathbb{R} \cup\{\infty,-\infty\}, \min , \max , \infty,-\infty)$,

3. $(K \cup\{\infty\}$, min, $\cdot, \infty, 1)$ for $K \in\left\{\mathbb{N}, \mathbb{Z}_{\geq 0}, \mathbb{Q}_{\geq 0}, \mathbb{R}_{\geq 0}\right\}$,

4. $([0,1] \cup\{\infty\}, \min , \cdot, \infty, 1)$,

5. $([1, \infty], \min , \cdot, \infty, 1)$,

6. $(K \cup\{-\infty\}, \max ,+,-\infty, 0)$ for $K \in\left\{\mathbb{N}, \mathbb{Z}_{\geq 0}, \mathbb{Q}_{\geq 0}, \mathbb{R}_{\geq 0}\right\}$,

7. $(K \cup\{\infty\}, \max , \min , 0, \infty)$ for $K \in\left\{\mathbb{N}, \mathbb{Z} \geq 0, \mathbb{Q} \geq 0, \mathbb{R}_{\geq 0}\right\}$,

8. $([0,1], \max , \min , 0,1)$,

9. $([0,1], \max , \cdot, 0,1)$,

or $\mathcal{K}$ is such that $(K, \odot, \mathbb{1})$ is locally finite, i.e., each finitely generated monoid is finite, then $\mathcal{T}^{-1}(k)$ is unambiguously recognizable.

Proof. First, we give the proof for $\left(\mathbb{R}_{\geq 0} \cup\{\infty\}\right.$, min, $\left.+, \infty, 0\right)$. The idea is as follows: assume that $N$ is the minimal weight occuring as a weight in a weighted timed automaton. Then, we only have to consider runs of length smaller than or equal to $\left\lceil\frac{k}{N}\right\rceil$. This is because every other run necessarily has a running weight greater than $k$. Thus, the set of occuring weights relevant for solving the problem is finite and can be remembered within the discrete part of a timed automaton.

Let $\mathcal{A}=\left(S, S_{0}, S_{f}, X, E, C\right)$ be a weighted timed automaton over $\mathcal{K}$ and $\mathcal{F}$ such that $\|\mathcal{A}\|=\mathcal{T}$ and $k \in \mathbb{R}_{\geq 0}$. We assume $C_{s}$ to be of the form $\min \left\{\alpha_{i}^{s}+\right.$ $\left.\chi_{A_{i}^{s}} \mid 1 \leq i \leq n_{s}\right\}$ for every $s \in S$. Define $N=\min (\operatorname{wgt}(\mathcal{A}) \backslash\{0\})$. Let $y$ be a "fresh" clock variable not occurring in $X$. We define the timed automaton $\mathcal{A}^{\prime}=\left(S^{\prime}, S_{0}^{\prime}, S_{f}^{\prime}, X^{\prime}, E^{\prime}\right)$ by

$$
\begin{aligned}
- & S^{\prime}=S \times\left\{m \in \mathbb{R}_{\geq 0} \cup\{\infty\} \mid m=m_{1}+\ldots+m_{p}, m_{1}, \ldots, m_{p} \in \operatorname{wgt}(\mathcal{A}), p \in\right. \\
& \left.\left\{1, \ldots,\left\lceil\frac{k}{N}\right\rceil\right\}\right\} \cup\{0, k\} \\
- & S_{0}^{\prime}=S_{0} \times\{0\} \\
- & S_{f}^{\prime}=S_{f} \times\{k\} \\
- & X^{\prime}=X \cup\{y\} \\
- & E^{\prime}=\left\{\left((s, m), a, \phi^{\prime}, \lambda^{\prime},\left(s^{\prime}, m^{\prime}\right)\right) \mid\left(s, a, \phi, \lambda, s^{\prime}\right) \in E \text { such that } \phi^{\prime}=\phi \wedge\right. \\
& \left.\left\langle A_{i}^{s}\right\rangle_{y}, \lambda^{\prime}=\lambda \cup\{y\}, m^{\prime}=m+\alpha_{i}+C_{\mathcal{E}}\left(\left(s, a, \phi, \lambda, s^{\prime}\right)\right) \text { for every } i \in\left\{1, \ldots, n_{s}\right\}\right\}
\end{aligned}
$$

Then, one can easily prove $L\left(\mathcal{A}^{\prime}\right)=\|\mathcal{A}\|^{-1}(k)$. The proof for $\mathbb{R}_{\geq 0}$ of course also works for $\mathbb{N}, \mathbb{Z}_{\geq 0}$ and $\mathbb{Q}_{\geq 0}$, which finishes the proof for 1 .

Now, let $\ln (x)$ denote the natural logarithm of a number $x$ and abbreviate $P_{\max }=([0,1], \max , \cdot, 0,1)$ and $\mathcal{R}_{\min }=\left(\mathbb{R}_{\geq 0} \cup\{\infty\}, \min ,+, \infty, 0\right)$. We define the semiring morphism $\eta:[0,1] \rightarrow \mathbb{R}_{>0} \cup\{\infty\}$ by $\eta(x)=-\ln (x)$. Let $\mathcal{T}$ be recognizable over $P_{\max }$ and $k \in[0,1] \backslash\{0\}$. Then, by Lemma $7, \eta \circ \mathcal{T}$ is recognizable over $\mathcal{R}_{\text {min }}$. Using 1., we know that $\left\{w \in T \Sigma^{*} \mid(\eta \circ \mathcal{T}, w)=\eta(k)\right\}$ is recognizable. But this implies that $\left\{w \in T \Sigma^{*} \mid(\mathcal{T}, w)=k\right\}$ is recognizable. This proves 8 .

Similar proof ideas can be used to prove the claim for the other semirings.

Lemma 9. Let $\mathcal{K}$ be a semiring such that $\odot=\min$ or $\odot=\max , \mathcal{F}$ is the family of linear functions of the form $c \cdot \delta$ such that $c \in \mathbb{Q}$ and $k \in \mathbb{Q}$. If $\mathcal{T}$ is an unambiguously recognizable timed series over $\mathcal{K}$ and $\mathcal{F}$, then $\mathcal{T}^{-1}(k)$ is unambiguously recognizable. 
Proof. We briefly summarize the idea of the construction of a timed automaton $\mathcal{A}$ recognizing $\|\mathcal{A}\|^{-1}(k)$. We need to remember whether during a run on a word we already have reached a weight of $k$ or not. We obtain this information by a newly introduced clock variable $y$ that measures the time that has been spent in the current location. At every edge a clock constraint on $y$ controls the behaviour of $\mathcal{A}$ depending on whether we have already reached a weight of $k$ or not beforehands, and on how much time we have already spent in the current location. This can be done owing to the restriction on $k$ being a rational number.

Lastly, we want to consider cut languages. Again, we can generalize results from the theory of weighted automata for recognizable timed series over the family of step functions owing to the finite number of weights occuring in a corresponding weighted timed automaton. Notice that in the next lemma, in opposition to Lemma 8, we do not require $\mathcal{T}$ to be unambiguous.

Lemma 10. Let $\mathcal{F}$ be the family of step functions, let $k \in K \backslash\{\mathbb{0}\}$ and $\mathcal{T}$ be a recognizable timed series. If $\mathcal{K}$ is one of the following semirings

1. $(K \cup\{\infty\}, \min ,+, \infty, 0)$ for $K \in\left\{\mathbb{N}, \mathbb{Z}_{\geq 0}, \mathbb{Q}_{\geq 0}, \mathbb{R}_{\geq 0}\right\}$,

2. $(\mathbb{R} \cup\{\infty,-\infty\}$, min, $\max , \infty,-\infty)$,

3. $(K \cup\{\infty\}, \min , \cdot, \infty, 1)$ for $K \in\left\{\mathbb{N}, \mathbb{Z}_{\geq 0}, \mathbb{Q}_{\geq 0}, \mathbb{R}_{\geq 0}\right\}$,

4. $([0,1] \cup\{\infty\}, \min , \cdot, \infty, 1)$,

5. $([1, \infty], \min , \cdot, \infty, 1)$,

then $\mathcal{T}^{-1}(\{m \mid m \leq k\}$ is recognizable. If $\mathcal{K}$ is one of the following semirings

1. $(K \cup\{-\infty\}, \max ,+,-\infty, 0)$ for $K \in\left\{\mathbb{N}, \mathbb{Z}_{\geq 0}, \mathbb{Q}_{\geq 0}, \mathbb{R}_{\geq 0}\right\}$,

2. $(K \cup\{\infty\}$, $\max , \min , 0, \infty)$ for $K \in\left\{\mathbb{N}, \mathbb{Z}_{\geq 0}, \mathbb{Q}_{\geq 0}, \mathbb{R}_{\geq 0}\right\}$,

3. $([0,1], \max , \min , 0,1)$,

4. $([0,1], \max , \cdot, 0,1)$,

then $\mathcal{T}^{-1}(\{m \mid m \geq k\}$ is recognizable.

Proof. We use the same approach as in the proof of Lemma 8. Notice that $\eta$ is an anti-isomorphism w.r.t. the natural orderings in $P_{\max }$ and $\mathcal{R}_{\geq 0}$. Thus, we have

$$
\left.\left\{w \in T \Sigma^{*} \mid(\eta \circ \mathcal{T}, w) \leq \eta(k)\right\}=\left\{w \in T \Sigma^{*} \mid(\mathcal{T}, w) \geq k\right)\right\}
$$

and can conclude that $\left.\left\{w \in T \Sigma^{*} \mid(\mathcal{T}, w) \geq k\right)\right\}$ is recognizable.

\section{Conclusion and Further Research}

In this paper, we investigated decidability problems concerning the supports (and subsets of supports, respectively) of recognizable timed series. We believe that this work is only the beginning of fruitful further research within this area, as there are a lot of open problems worth considering. For instance, some results of this paper do not cover recognizable timed series over certain important semirings and families of cost functions. In particular, we would like to know whether 
there is a recognizable timed series over $\left(\mathbb{R}_{\geq 0},+, \cdot, 0,1\right)$ and the family of linear functions such that $\mathcal{T}^{-1}(0)$ is not recognizable. Also, we are interested in what happens if we let $\mathcal{F}$ contain both linear and step functions. This is needed to model so-called stopwatch automata, where the cost variable can be switched on in some locations (the cost functions are linear) and switched out in the other locations (the cost functions map every time delay to the constant $\mathbb{1}$ ). However, we cannot apply the proof method of e.g. Lemma 4 when $\mathcal{F}$ contains both linear and step functions. Of course, one may think of cost functions other than the step or linear cost functions as e.g. functions of the form $k \cdot \ln (\delta), k^{\delta}$ etc., and other semirings. Also, there are lots of interesting applications that require operations that do not form a semiring and have recently been introduced under the notion of quantitative languages [15]. This model is promising also within the context of real-time systems and has in fact already been used [11,19]. Thus we want to consider it in future work. Last but not least, for those settings where the supports (cut languages, respectively) of recognizable timed series in general are not guaranteed to be recognizable, we want to further examine whether we can decide whether a particular timed series has a recognizable support (cut language, respectively) or not. Altogether, with this work we hope to give a new perspective on the work on weighted timed automata.

\section{References}

1. Y. Abdeddaïm and O. Maler. Preemptive job-shop scheduling using stopwatch automata. In TACAS, volume 2280 of $L N C S$, pages 113-126. Springer, 2002.

2. R. Alur and D. L. Dill. A theory of timed automata. Theoretical Computer Science, 126(2):183-235, 1994.

3. R. Alur, S. La Torre, and G. J. Pappas. Optimal paths in weighted timed automata. In $H S C C$, volume 2034 of $L N C S$, pages 49-62. Springer, 2001.

4. R. Alur and P. Madhusudan. Decision problems for timed automata: A survey. In $S F M-R T$, volume 3185 of $L N C S$, pages 1-24. Springer, 2004.

5. G. Behrmann and A. Fehnker. Efficient guiding towards cost-optimality in UPPAAL. In TACAS, volume 2031 of $L N C S$, pages 174-188. Springer, 2001.

6. G. Behrmann, A. Fehnker, T. Hune, K. Larsen, P. Pettersson, J. Romijn, and F. Vaandrager. Minimum-cost reachability for priced timed automata. In $H S C C$, volume 2034 of $L N C S$, pages 147-161. Springer, 2001.

7. J. Berstel and C. Reutenauer. Rational series and their languages. Current online version of the book of the same name from 1988. February 2007.

8. P. Bouyer. Weighted timed automata: Model-checking and games. In MFPS'06, volume 158 of ENTCS, pages 3-17. Elsevier Science Publishers, 2006.

9. P. Bouyer, T. Brihaye, V. Bruyère, and J.-F. Raskin. On the optimal reachability problem on weighted timed automata. Formal Methods in System Design, 31(2):135-175, October 2007.

10. P. Bouyer, T. Brihaye, and N. Markey. Improved undecidability results on weighted timed automata. Inf. Process. Lett., 98(5):188-194, 2006.

11. P. Bouyer, E. Brinksma, and K. G. Larsen. Optimal infinite scheduling for multipriced timed automata. Formal Methods in System Design, 32(1):3-23, 2008.

12. P. Bouyer and N. Markey. Costs are expensive! In FORMATS, volume 4763 of LNCS, pages 53-68. Springer, 2007. 
13. T. Brihaye, V. Bruyère, and J.-F. Raskin. On optimal timed strategies. In FORMATS, volume 3829 of LNCS, pages 49-64. Springer, 2005.

14. T. Brihaye, V. Bruyère, and J.-F. Raskin. On model-checking timed automata with stopwatch observers. Inf. Comput., 204(3):408-433, 2006.

15. K. Chatterjee, L. Doyen, and T. A. Henzinger. Quantitative languages. In CSL, volume 5213 of $L N C S$, pages 385-400. Springer, 2008.

16. M. Droste and K. Quaas. A Kleene-Schützenberger Theorem for Weighted Timed Automata. In FoSSaCS, volume 4962 of $L N C S$, pages 142-156. Springer, 2008.

17. I. Fichtner. Characterizations of Recognizable Picture Series. PhD thesis, Universität Leipzig, Institut für Informatik, Abteilung Automaten und Sprachen, 2006.

18. D. Kirsten. The support of a recognizable series over a zero-sum free, commutative semiring is recognizable. In DLT, volume 5583 of $L N C S$, pages $326-333$. Springer, 2009.

19. K. G. Larsen and J. I. Rasmussen. Optimal reachability for multi-priced timed automata. Theor. Comput. Sci., 390(2-3):197-213, 2008.

20. J. Ouaknine and J. Worrell. On the Language Inclusion Problem for Timed Automata: Closing a Decidability Gap. In LICS, pages 54-63. IEEE Computer Society Press, 2004.

21. K. Quaas. Weighted Timed MSO Logics. In DLT, volume 5583 of $L N C S$, pages 419-430. Springer, 2009.

22. G. Rahonis. Fuzzy languages. In Droste, Kuich, Vogler, editors. Handbook of Weighted Automata. Springer, 2009. To appear.

23. J. Sakarovitch. Rational and recognisable power series. In Droste, Kuich, Vogler, editors. Handbook of Weighted Automata. Springer, 2009. To appear.

24. A. Salomaa and M. Soittola. Automata-Theoretic Aspects of Formal Power Series. Springer New York, 1978.

25. T. Wilke. Automaten und Logiken zur Beschreibung zeitabhängiger Systeme. PhD thesis, Christian-Albrecht-Universität Kiel, 1994. 